ADDITIONAL MATERIALS

\title{
Natural Gas: From Shortages to Abundance in the U.S.
}

\author{
Paul L. Joskow
}

January 4, 2013 


\section{SUMMARY}

- Tortured history of natural gas wellhead price and interstate gas pipeline regulation, deregulation and regulatory reform 1938-1993: creating and responding to natural gas shortages and then contract surpluses 1970-1990

- Deregulation of wellhead prices, unbundling, and pipeline regulatory reform ultimately created a platform conducive to efficient static and dynamic performance on the supply and demand sides of the natural gas market

- Shale gas resources became economical to exploit due to technological innovations in drilling technologies that could easily be exploited without the burdens of inefficient economic regulation

- The magnitude of shale gas production was a "surprise". Early 2000's expectation was that U.S. would become a large importer of natural gas from Canada, Alaska, LNG from Middle East and North Africa.

- Expected North American production of shale gas keeps increasing year after year, gas prices have fallen significantly, but are probably below long run equilibrium levels, and the U.S. is now expected to be a gas exporter by about 2020 .

- Natural gas consumers will benefit from lower gas prices, the trajectory of CO2 emissions will be lower, and there are national security benefits from going from an expected gas importer to an expected gas exporter

- Unresolved environmental concerns, along with misleading information and a lack of transparency, have led to public opposition in some areas. These issues can be and must be resolved if shale gas is to reach its full potential 
U.S. Natural Gas Wellhead Price

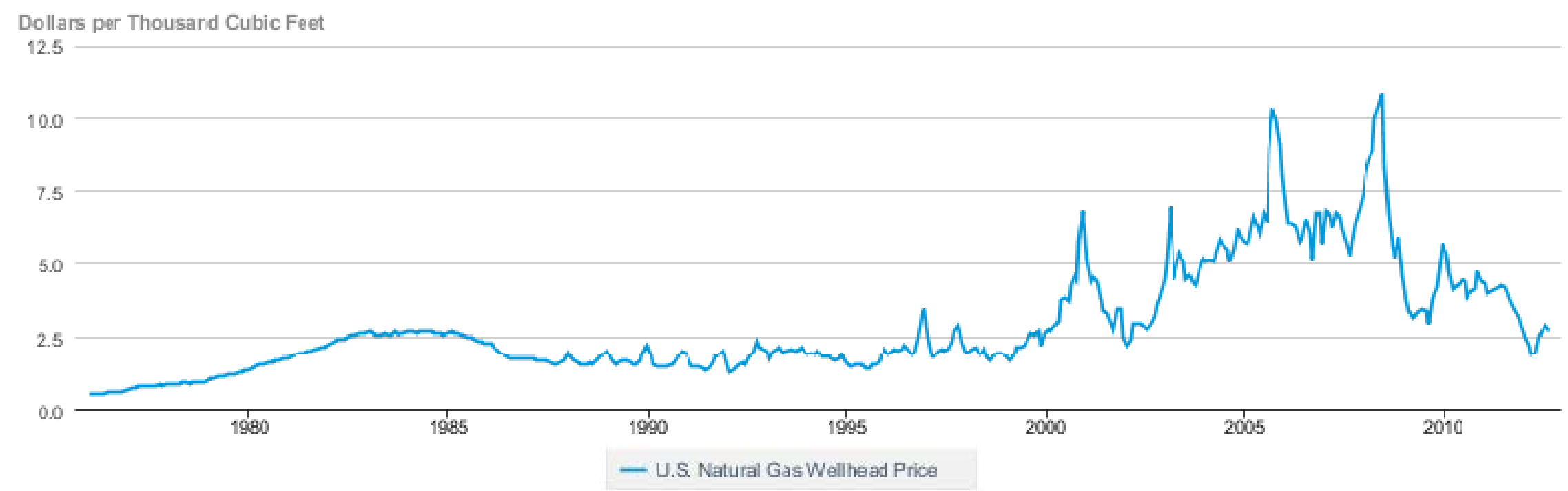

อ1ล Source: U.S. Energy information Aaminsiration

Source: U.S. ElA http://www.eia.gov/dnav/ng/hist/n9190us3m.htm 12/26/12 


\section{Natural Gas Regulation, Deregulation and Regulatory Reform}

- 1938 --- FPC pipeline regulation of bundled gas and pipeline services

- 1954 --- Phillips Decision: FPC Regulation of wellhead prices begins

- $1954-1979$--- Various approaches to cost of service regulation applied to set maximum wellhead prices.

- Blending and bundling of gas contracts by pipelines key to implementation

- 1970 - 1981 --- Shortages and rationing

- 1978: Natural Gas Policy Act: old gas, new gas, high cost gas ; category by category ceiling prices.

- Pipelines rush to sign long term contracts for "new gas"with high ceiling prices and "blend" diverse contract prices together to create average costbased sale prices

- 1981: Oil prices peak and then fall $2 / 3$ by 1986 . "Take or pay" contract surpluses. 


\section{Natural Gas Regulation, Deregulation and Regulatory Reform}

- 1981-85: SMPs for customers with fuel switching capabilities and the start of unbundling. Clear price discrimination leads to rejection by the courts

- 1985: FERC Order 436 voluntary unbundling available to all customers

- 1987: FERC Order 500 and take or pay contract renegotiation

- 1989: Natural Gas Wellhead Decontrol Act

- 1992: FERC Order 636 makes unbundling mandatory

- 1993: Regulation of wellhead prices formally ends

- 1990s: Competitive natural gas markets and light handed regulation of pipeline services mature --- prices low and stable

- 2000: (ex post) "Gas bubble" ends, gas prices rise and become volatile.

- 2001-2006: Large increase in pipeline imports from Alaska, Canada and LNG imports from other countries and higher prices expected to balance U.S. supply and demand in the future

- 2007: Shale gas slowly begins to be recognized as important future supply source

- 2009: Shale gas production starts to have significant effect on domestic gas supplies leading to much lower gas prices

- 2011: LNG exports begin to get serious attention in the U.S. and Canada 
Figure 20. Major sources of incremental natural gas supply, 2002-2025 (trillion cubic feet)

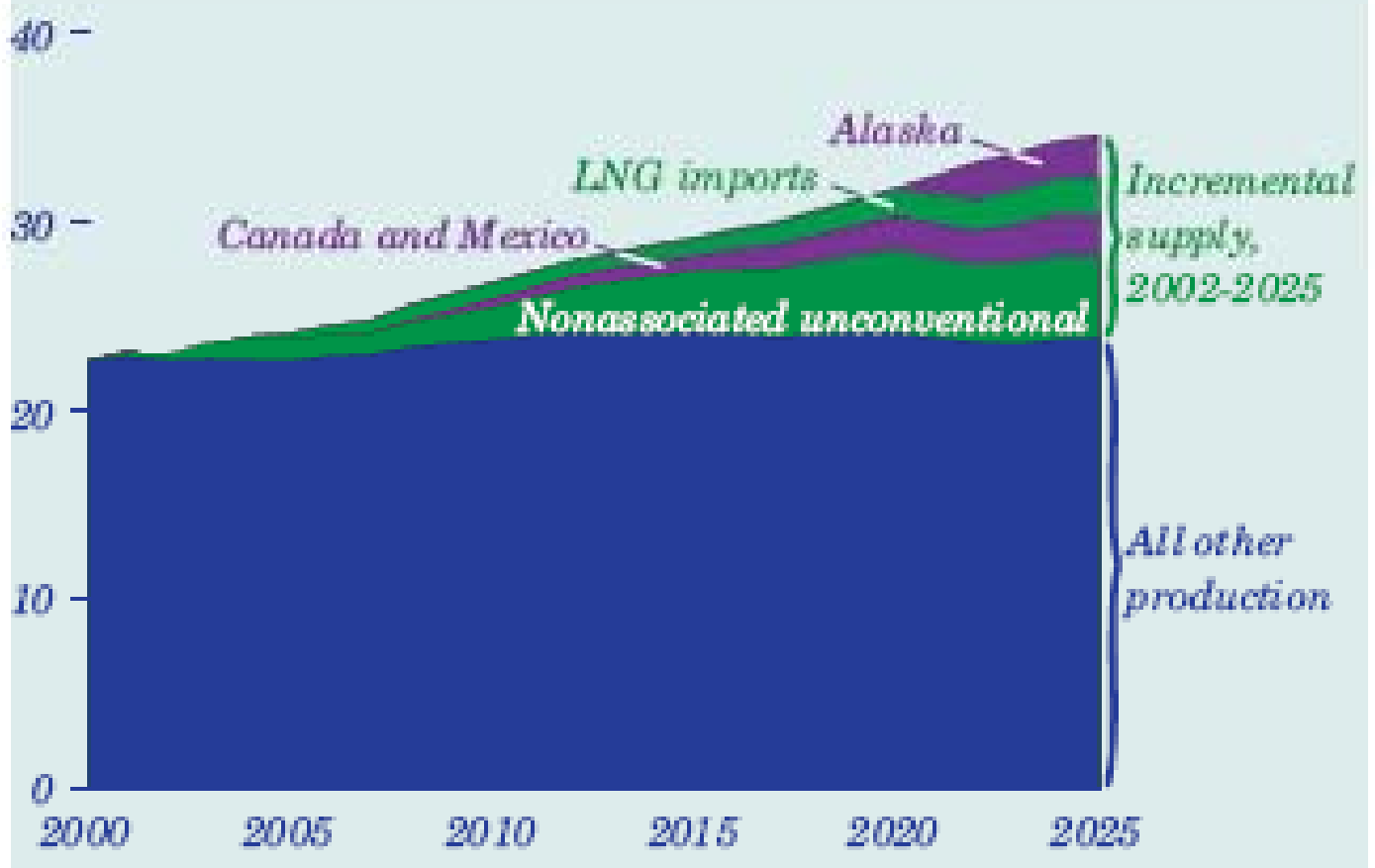

EIA AEO 2003 


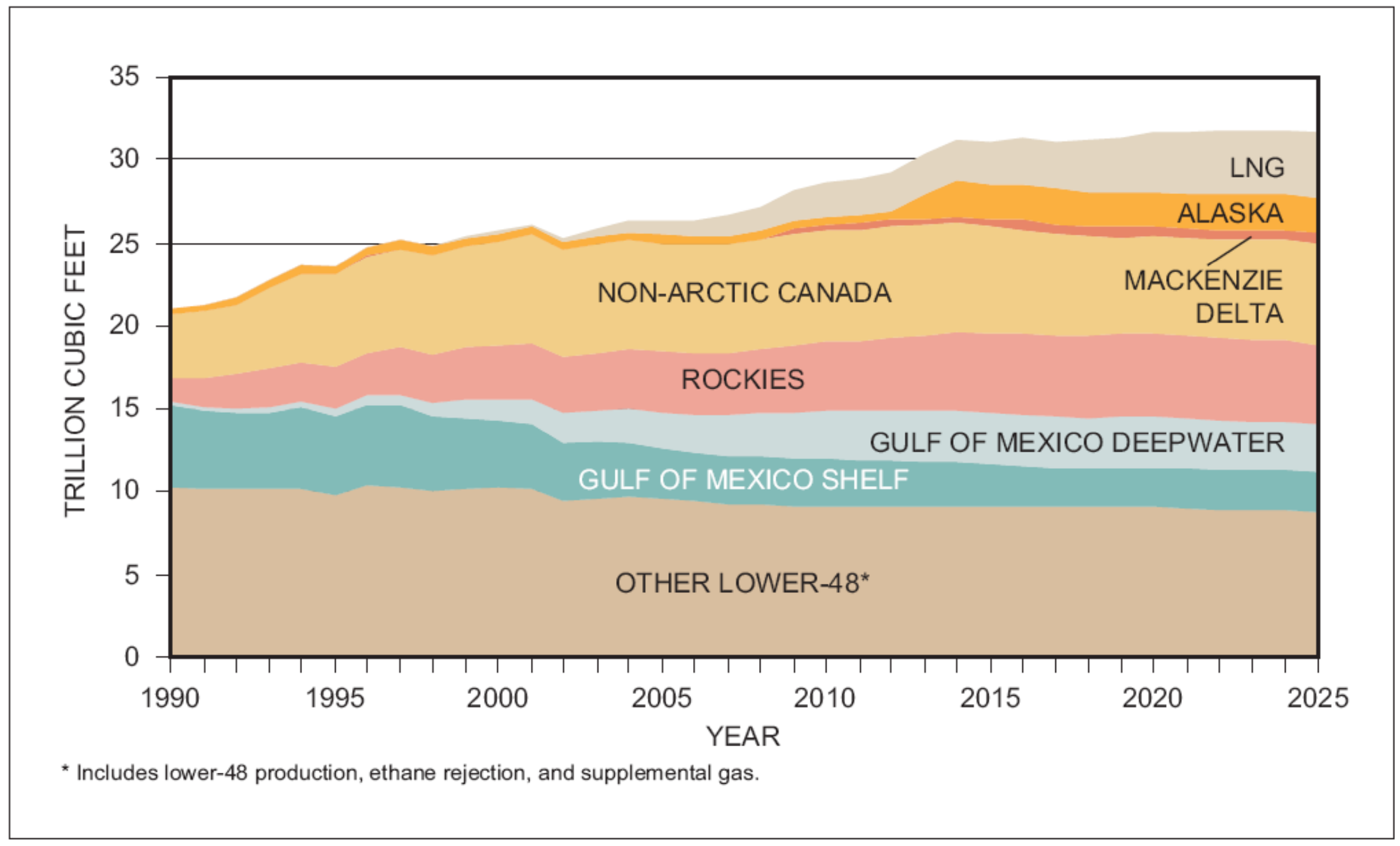

Figure 38. U.S. and Canadian Natural Gas Supply

NPCC 2003 
Figure 14. Lower 48 natural gas production by resource type, 1990-2025 (trillion cubic feet)

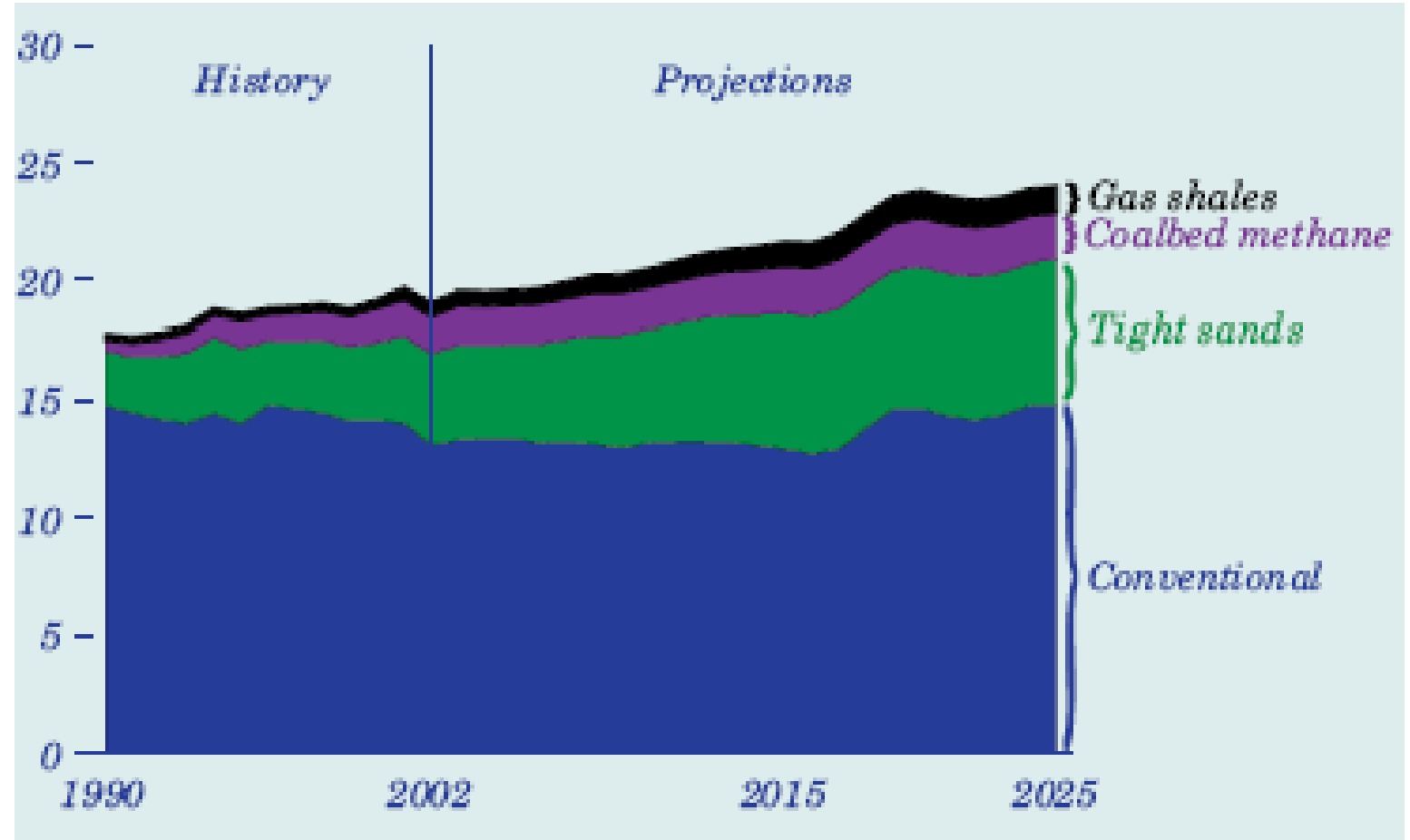

EIA AEO 2004 
Figure 17. Major sources of incremental natural gas supply, 2002-2025 (trillion cubic feet)

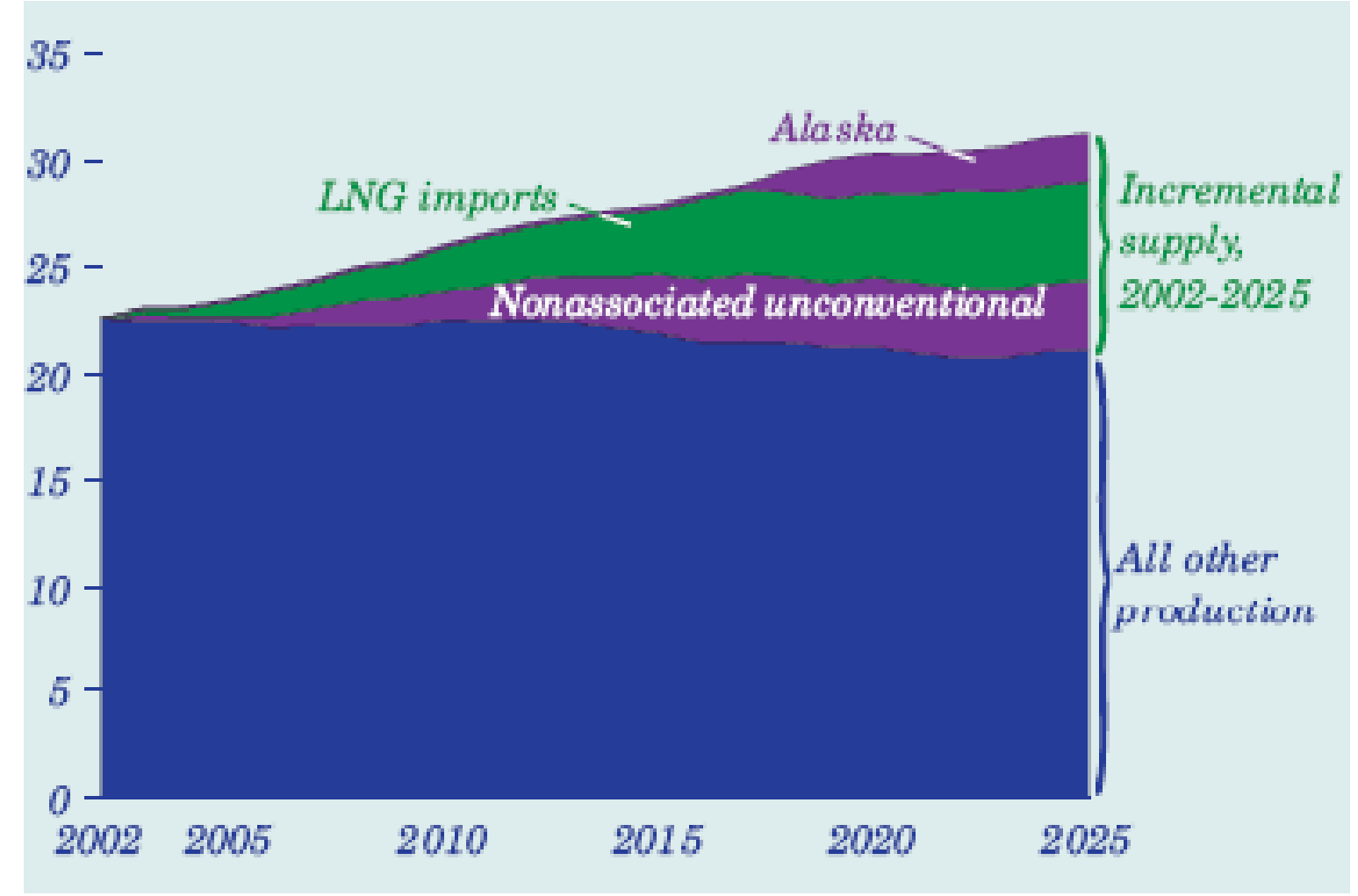

EIA AEO 2004 
Figure 21. Projected LNG imports by terminal and region in the reference case, 2025 (billion cubic feet)

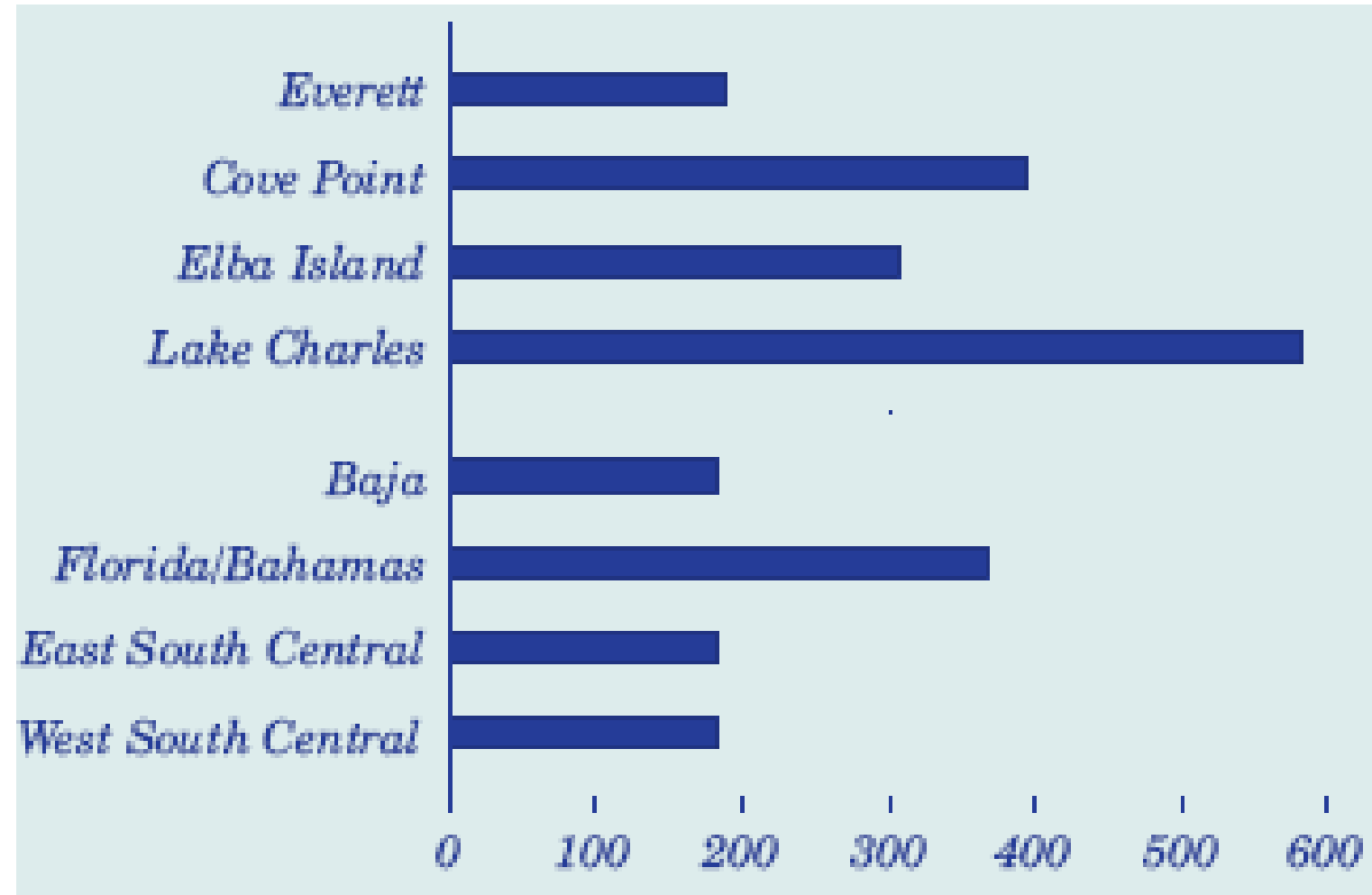

EIA AEO 2004 
Table 12. North American LNG regasification proposals as of December 1, 2003 (million cubic feet per day)

\begin{tabular}{|c|c|c|c|c|}
\hline Project & Owners & Location & $\begin{array}{l}\text { Start } \\
\text { year }\end{array}$ & $\begin{array}{c}\text { Capacity } \\
\text { added }\end{array}$ \\
\hline \multicolumn{5}{|l|}{ West Coast } \\
\hline Terminal GNL Mar Adentro de B.C. & CheuronTexaco & Baja California, Mexico (offshore) & 2007 & 750 \\
\hline Tijuana Regional Energy Center & Marathon/Golar LNG/Grupo GGS & Baja California, Mexico & 2006 & 750 \\
\hline Sound Energy Solutions & Mitsubishi & Long Beach, California & 2007 & 700 \\
\hline Terminal LNG de Baja California & Shell & Baja California, Mexico & 2007 & 1,000 \\
\hline Energia Costa Azul LNG & Sempra Energy & Baja California, Mexico & 2007 & 1,000 \\
\hline Crystal & Crystal Energy & Oxnard, California (offshore) & 2006 & 600 \\
\hline Tractebel Mexico & Tractebel & Lazaro Cardenas, Mexico & 2007 & 500 \\
\hline Cabrillo Port LNG & BHP Billiton & Oxnard, California (offshore) & 2008 & 1,500 \\
\hline \multicolumn{5}{|l|}{ Florida/Bahamas } \\
\hline Ocean Express LNG & AES & Ocean Cay, Bahamas & 2006 & 850 \\
\hline Freeport & El Paso & Freeport Grand Island, Bahamas & 2007 & 500 \\
\hline Calypso & Tractebel Bahamas LNG & Freeport Grand Cayman, Bahamas & 2007 & 832 \\
\hline \multicolumn{5}{|l|}{ Gulf Coast } \\
\hline ExxonMobil LNG & ExxonMobil & Quintana Island, Texas & 2007 & 1,000 \\
\hline Sabine Pass/Cheniere & Cheniere & Sabine Pass, Texas & 2008 & 2,000 \\
\hline Port Pelican & ChevronTexaco & Louisiana (offshore) & 2007 & 1,600 \\
\hline Cameron $L N G$ & Sempra Energy & Hackberry, Louisiana & 2007 & 1,500 \\
\hline Altamira & Shell & Altamira, Mexico & 2004 & 500 \\
\hline Corpus Christi LNG & Cheniere Energy & Corpus Christi, Texas & 2008 & 2,000 \\
\hline ExxonMobil/Sabine Pass LNG & ExxonMobil & Sabine Pass, Texas & 2008 & 1,000 \\
\hline Liberty & HNG Storage/Conversion Gas & Cameron, Louisiana & 2007 & 3,000 \\
\hline Main Pass Energy Hub & Freeport-McMoRan Sulphur & Gulf of Mexico (offshore) & 2006 & 1,500 \\
\hline Gulf Landing & Shell & West Cameron, Louisiana (offshore) & 2008-2009 & 1,000 \\
\hline Vermilion 179 & Conversion Gas Imports & Louisiana & 2008 & 1,000 \\
\hline Mobile Bay LNG & ExxonMobil & Mobile Bay, Alabama & 2008 & 1,000 \\
\hline Freeport LNG & Freeport, Cheniere, Contango & Freeport, Texas & 2006 & 1,500 \\
\hline Energy Bridge & El Paso & Floating Dock (offshore) & 2005 & 500 \\
\hline \multicolumn{5}{|l|}{ East Coast } \\
\hline Canaport & Irving Oil/Chevron Texaco & Canaport, New Brunswick, Canada & 2006 & 500 \\
\hline Weaver's Cove & Poten & Fall River, Massachusetts & 2007 & 400 \\
\hline Access Northeast Energy & Access Northeast Energy & Bearhead, Nova Scotia, Canada & 2008 & 500 \\
\hline Fairwinds LNG & TransCanada, ConocoPhillips & Harpswell, Maine & 2009 & 500 \\
\hline Providence $L N G$ & Keyspan, BG LNG Services & Providence, Rhode Island & 2005 & 500 \\
\hline Crown Landing & $B P$ & Logan Township, New Jersey & 2008 & 1,200 \\
\hline Somerset $L N G$ & Somerset $L N G$ & Somerset, Massachusetts & 2007 & 430 \\
\hline
\end{tabular}

\section{EIA AEO 2004}


Figure 20. U.S. net imports of LNG and Canadian natural gas, 1990-2025 (trillion cubic feet)

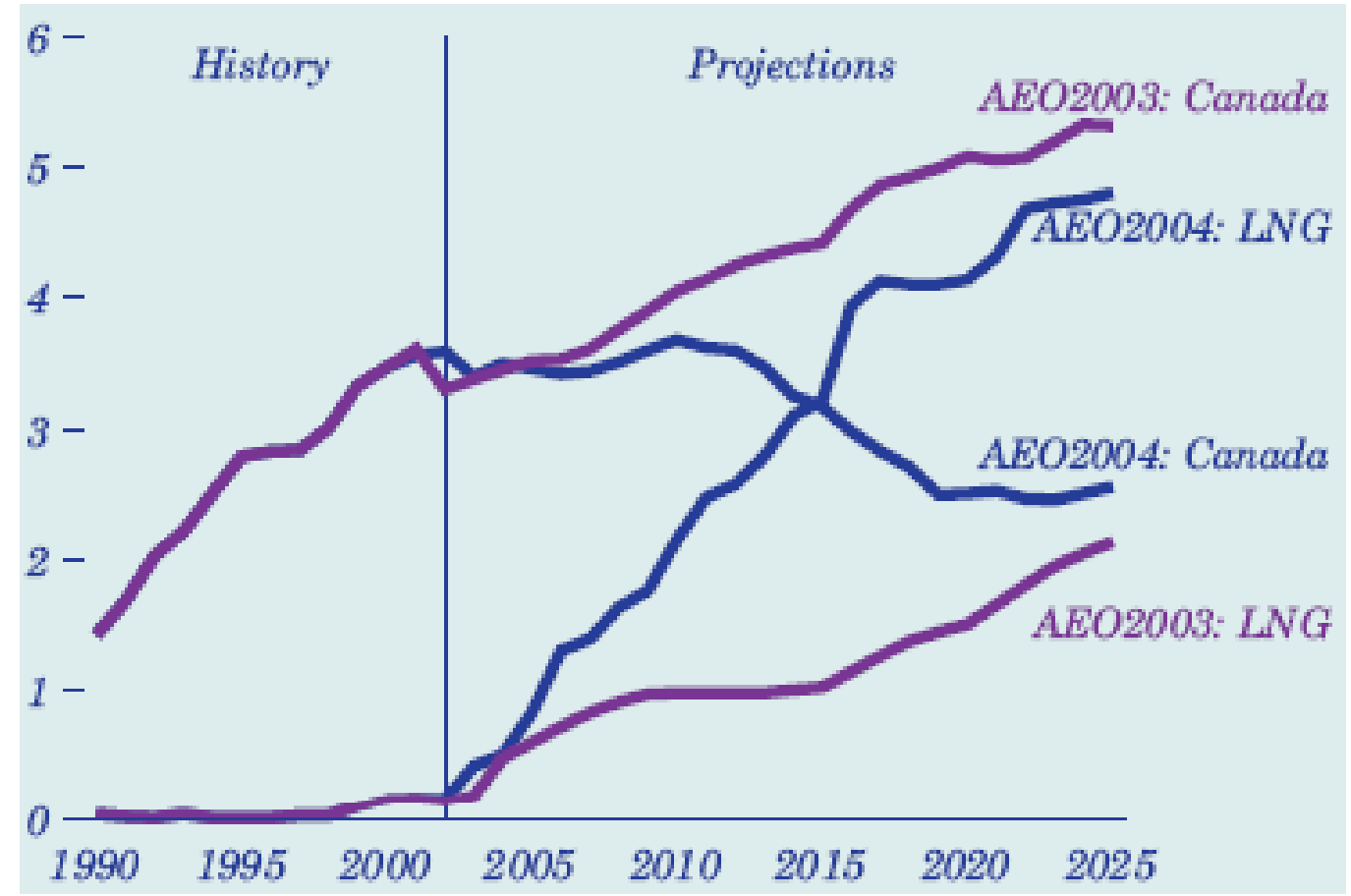

EIA AEO 2004 


\section{North American shale plays}

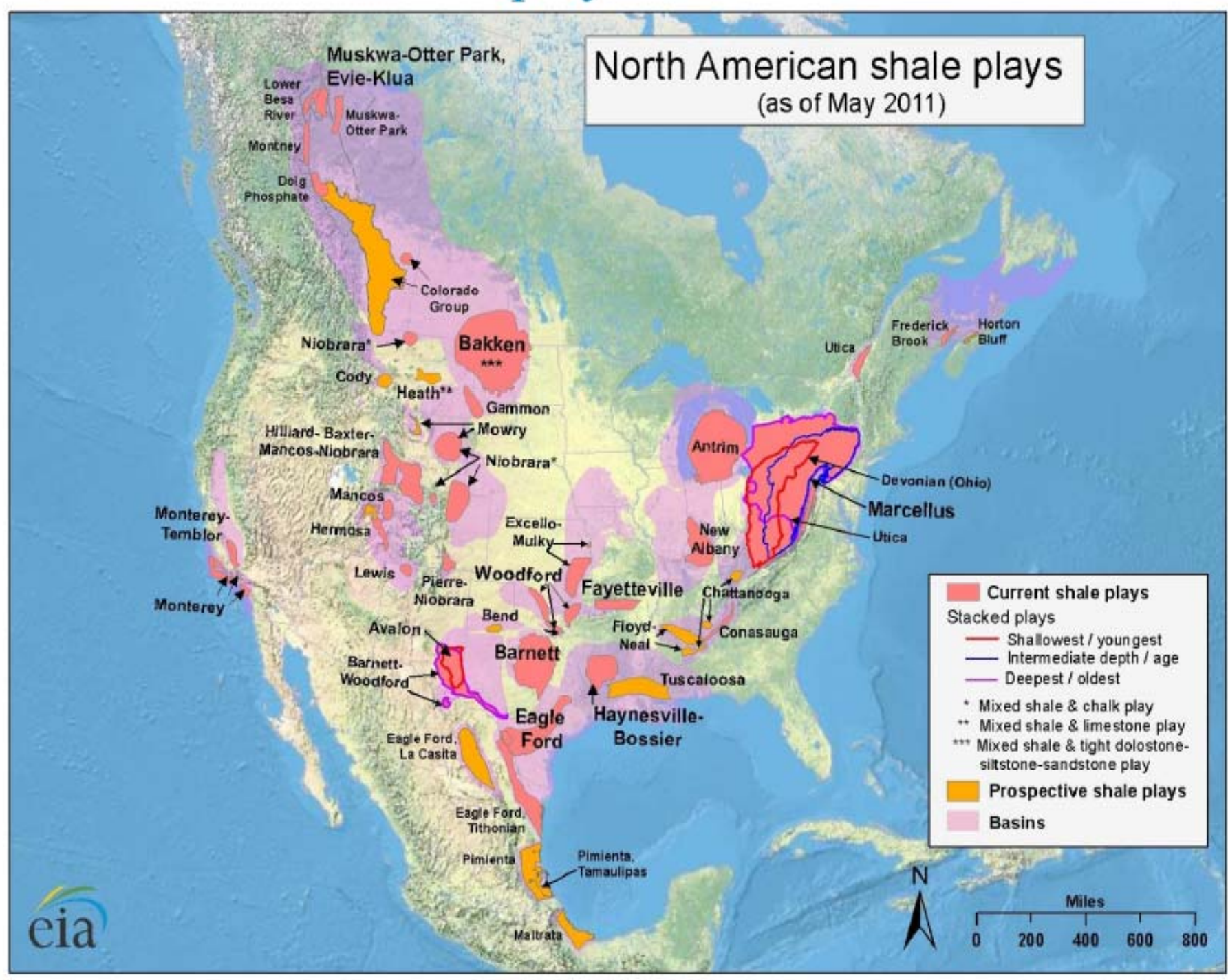

Source: U.S. Energy Information Administration based on data from various published studies. Canada and Mexico plays from ARI. 


\section{Underground sources of natural gas}

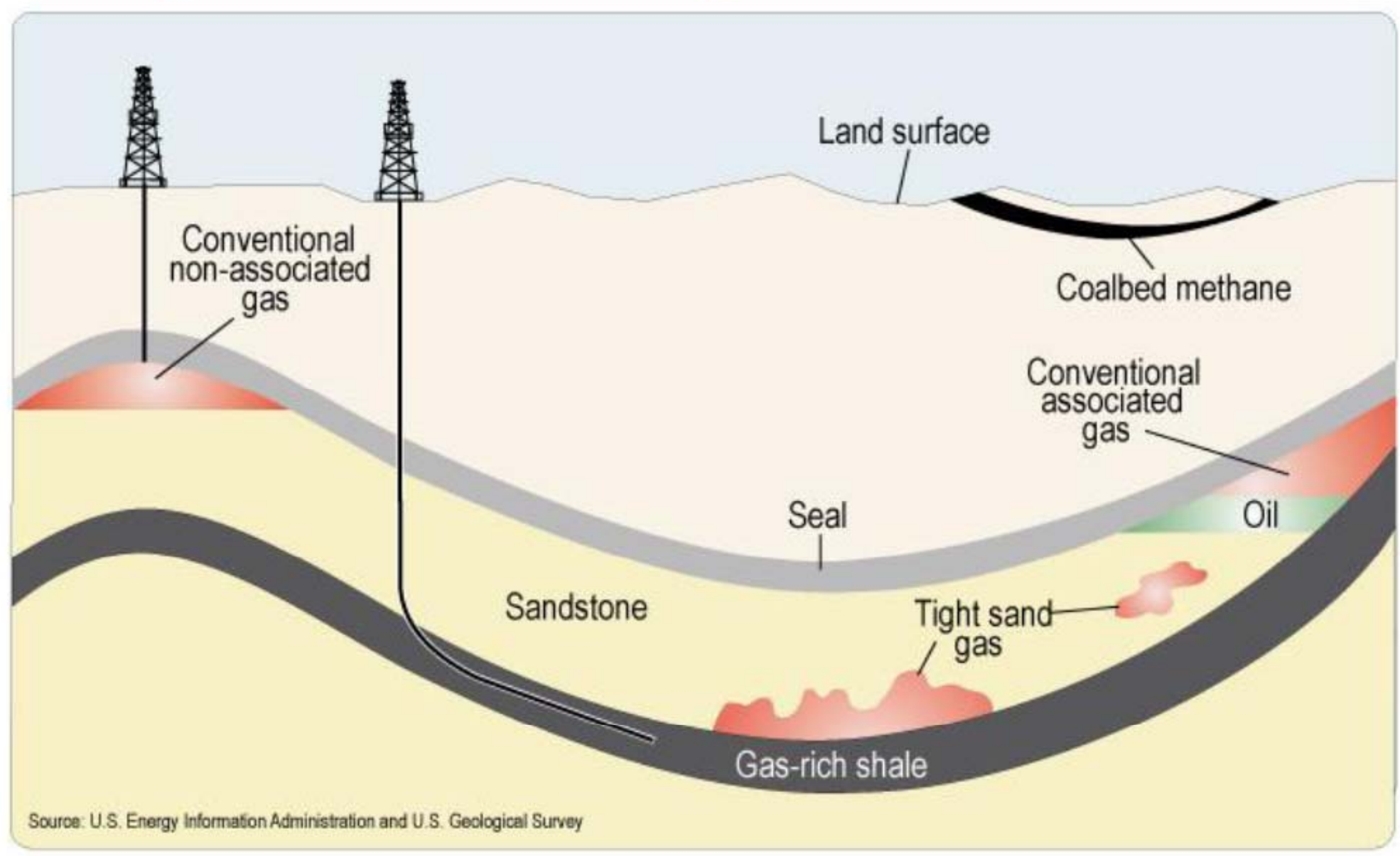

Source: modified from U.S. Geological Survey Fact Sheet 0113-01 


\section{Diagram of a typical hydraulic fracturing operation}

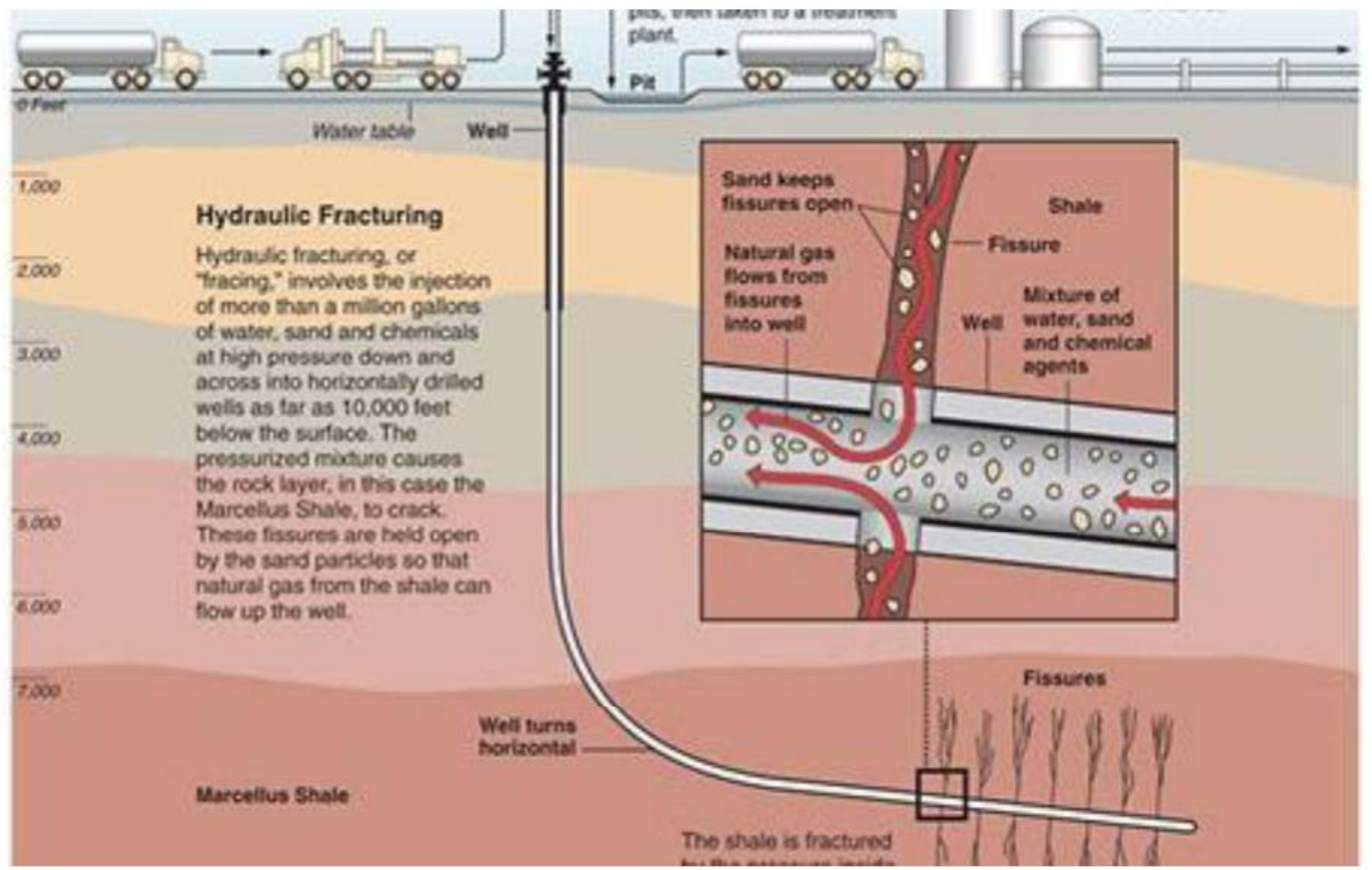

Source: ProPublica, http://www.propublica.org/special/hydraulic-fracturing-national 
Figure 3. U.S. dry natural gas production by source, 1990-2040 (trillion cubic feet)

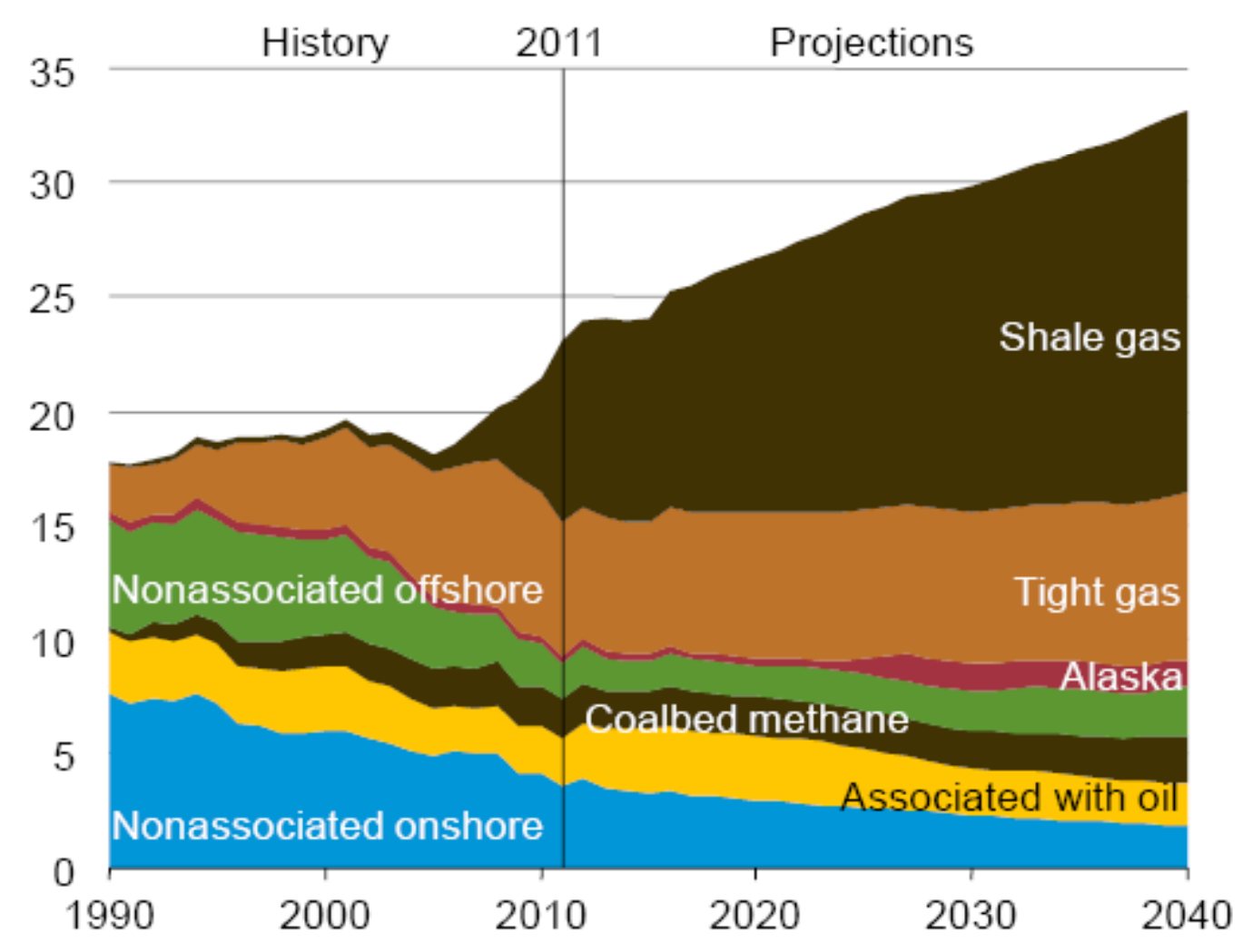

EIA AEO 2013 Early Release (December 2012) 
Figure 12. Electricity generation by fuel, 1990-2040 (trillion kilowatthours per year)

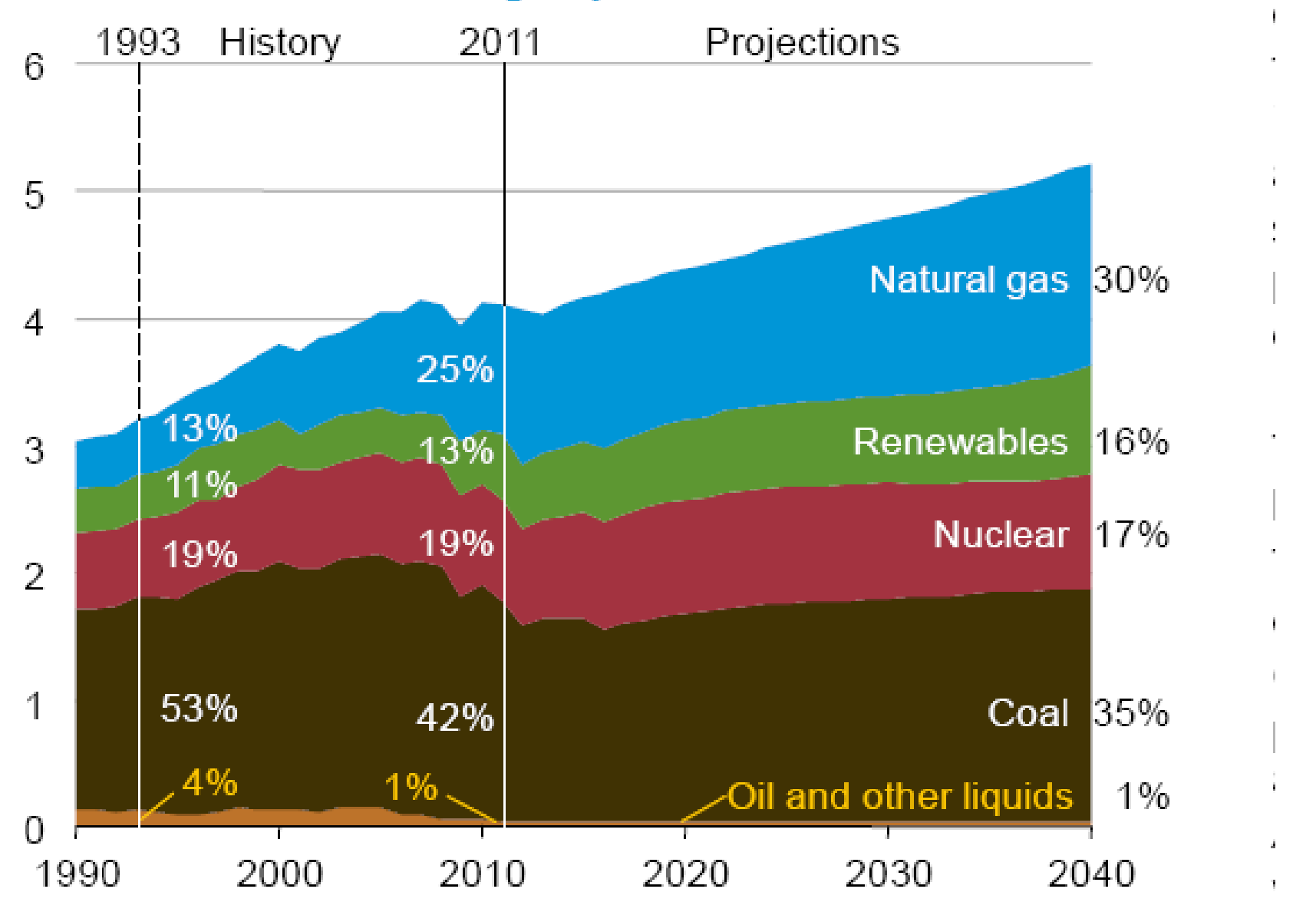


Figure 6. Delivered energy consumption by sector; 1980-2040 (quadrillion Btu)

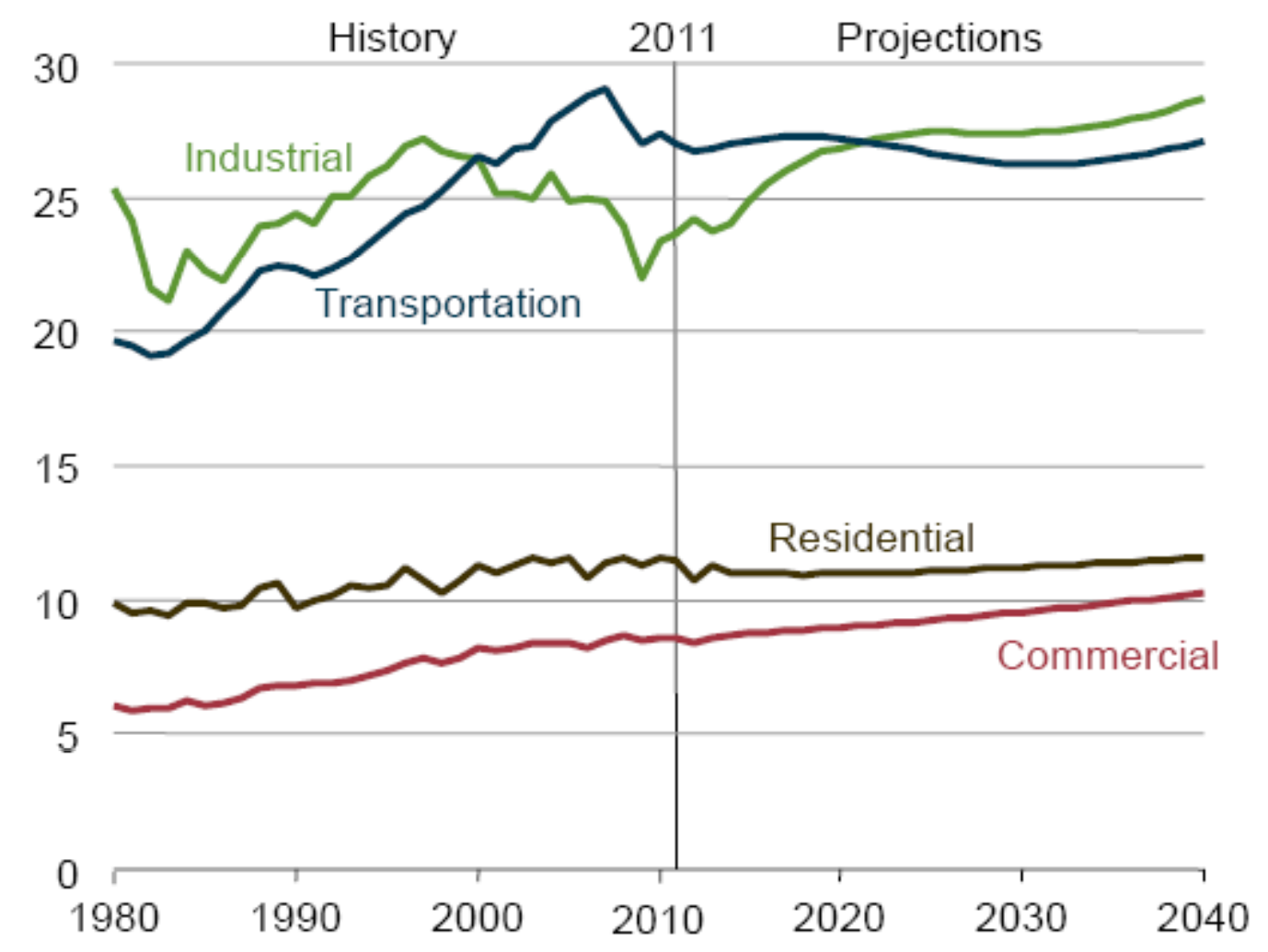

AEO 2013 Early Release 
Figure 4. Total U.S. natural gas production, consumption, and net imports, 1990-2035 (trillion cubic feet)

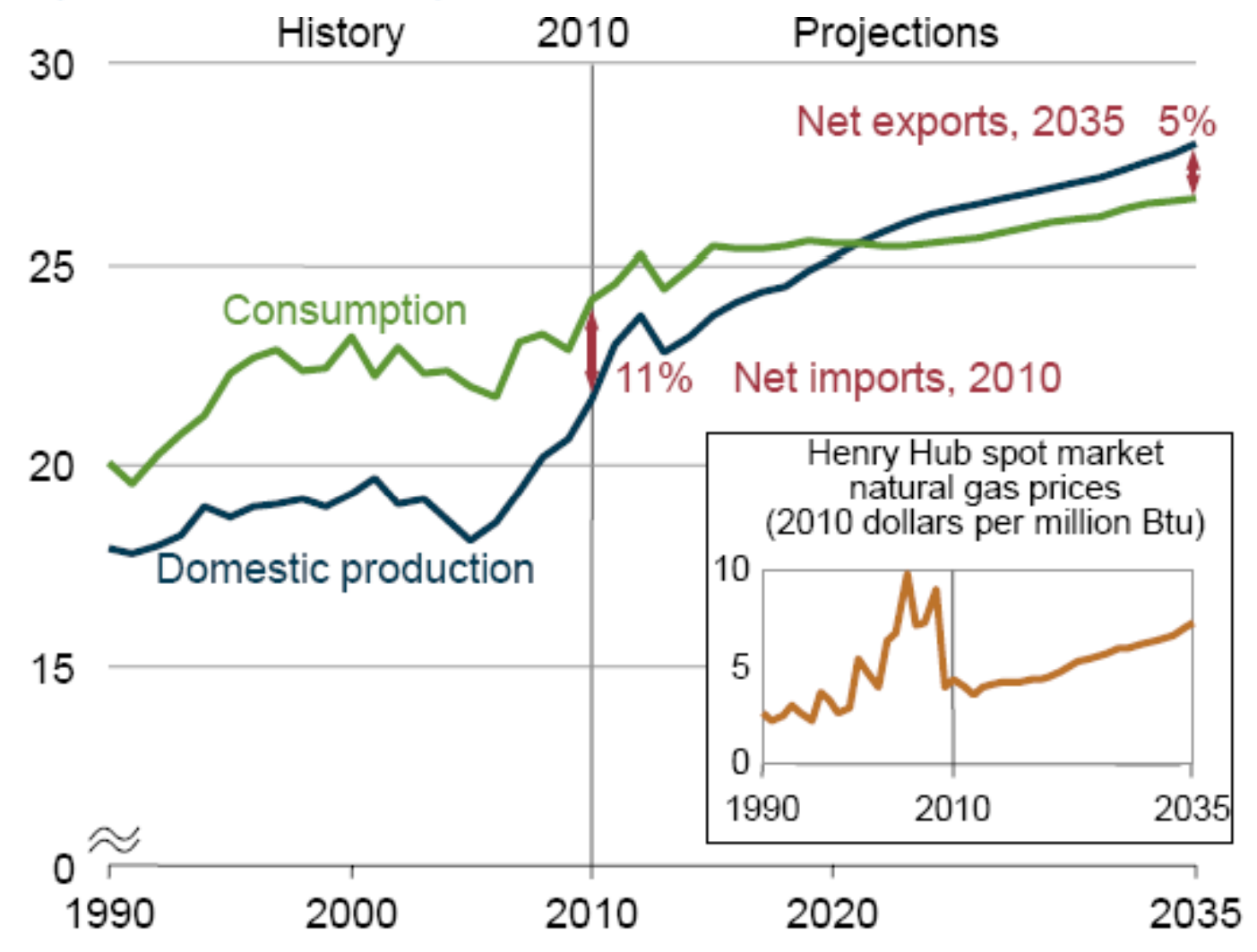

Source: EIA AEO 2012 


\section{The U.S. becomes a net natural gas exporter}

Figure 109. U.S. net imports of natural gas by source, 1990-2035 (trillion cubic feet)

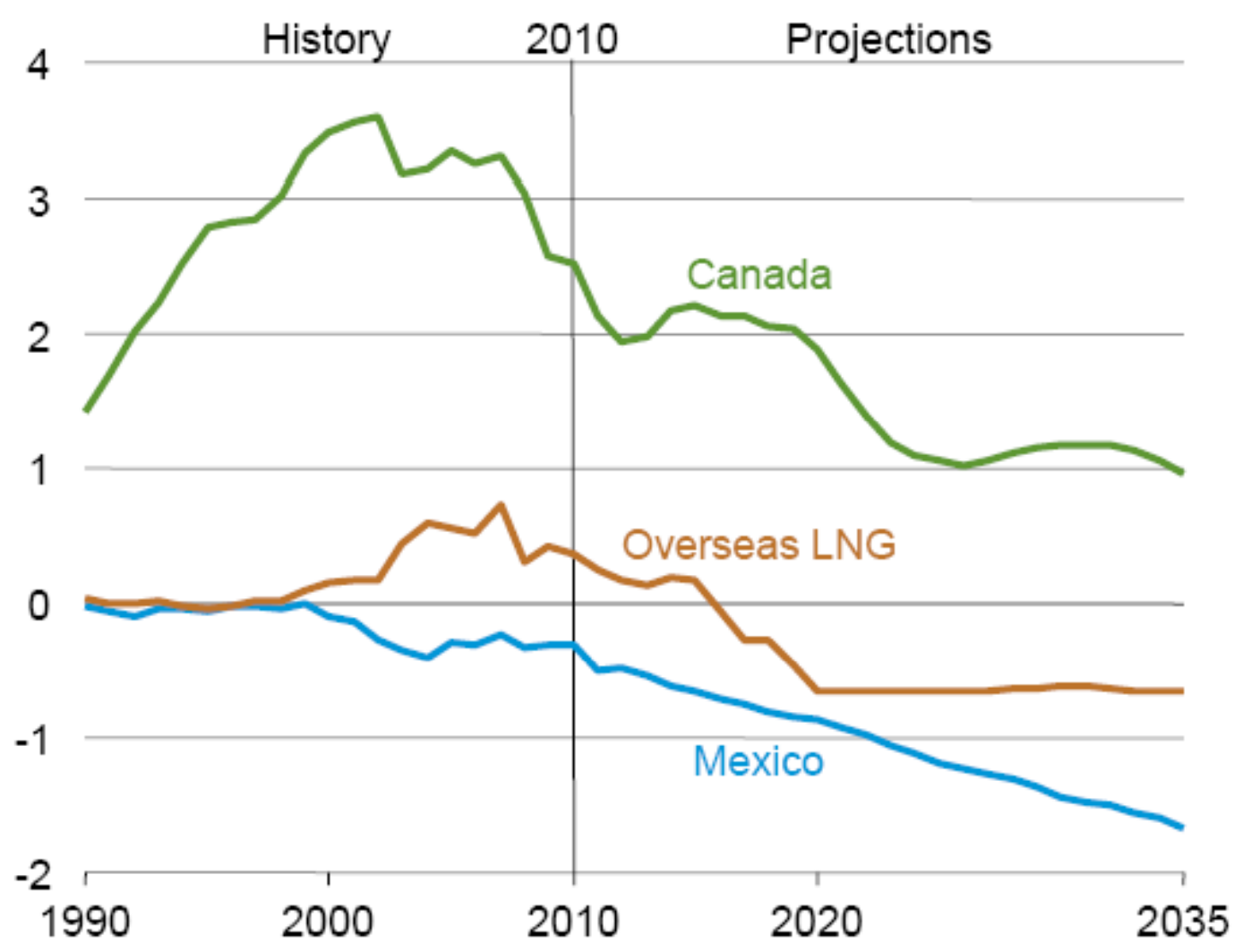

Source: EIA AEO 2012 
U.S. crude oil production increases, led by lower 48 onshore production

Figure 112. Domestic crude oil production by source, 1990-2035 (million barrels per day)

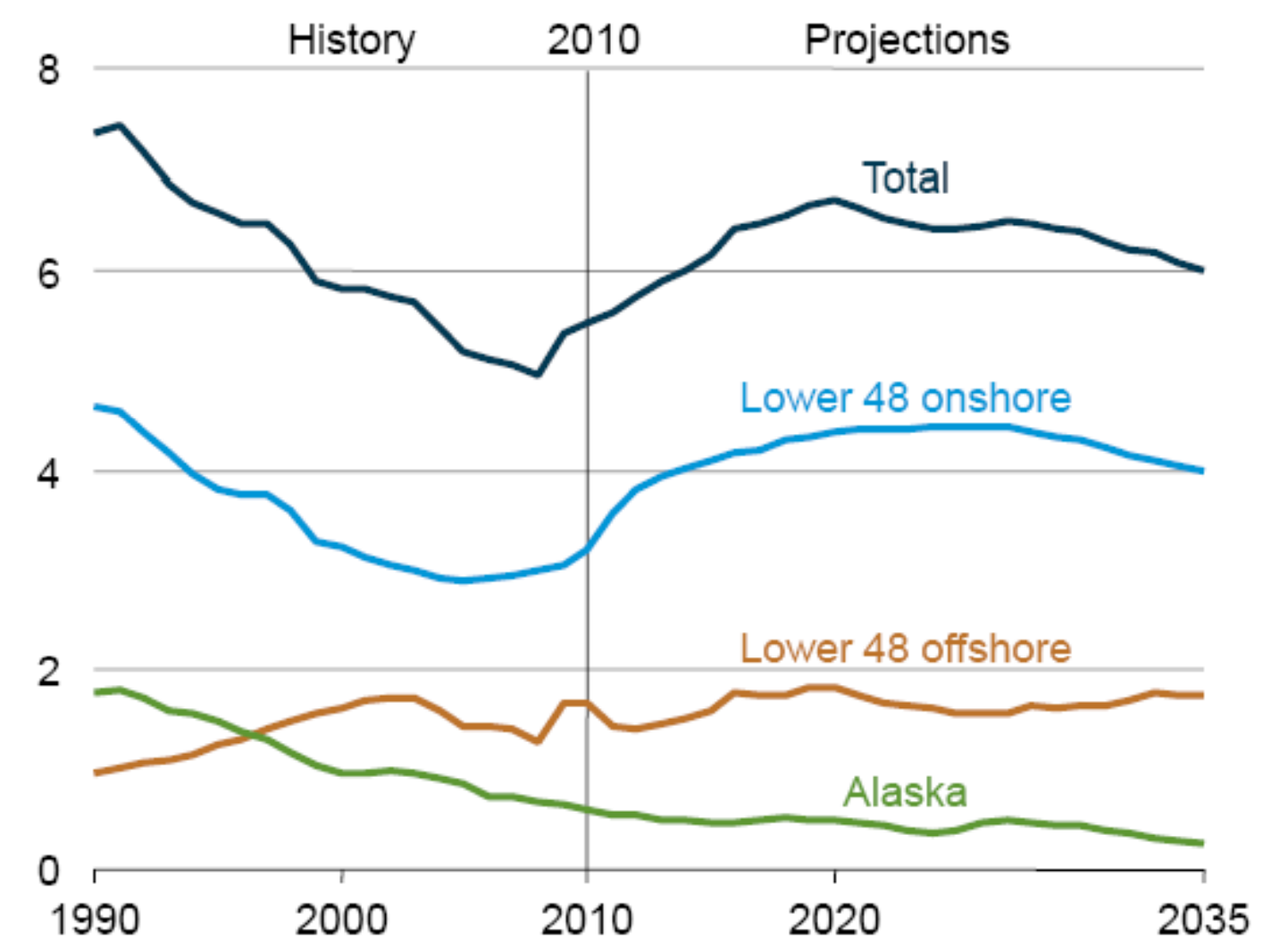

Source: EIA AEO 2012 
Figure 7. U.S. primary energy consumption by fuel, 1980-2040 (quadrillion Btu per year)

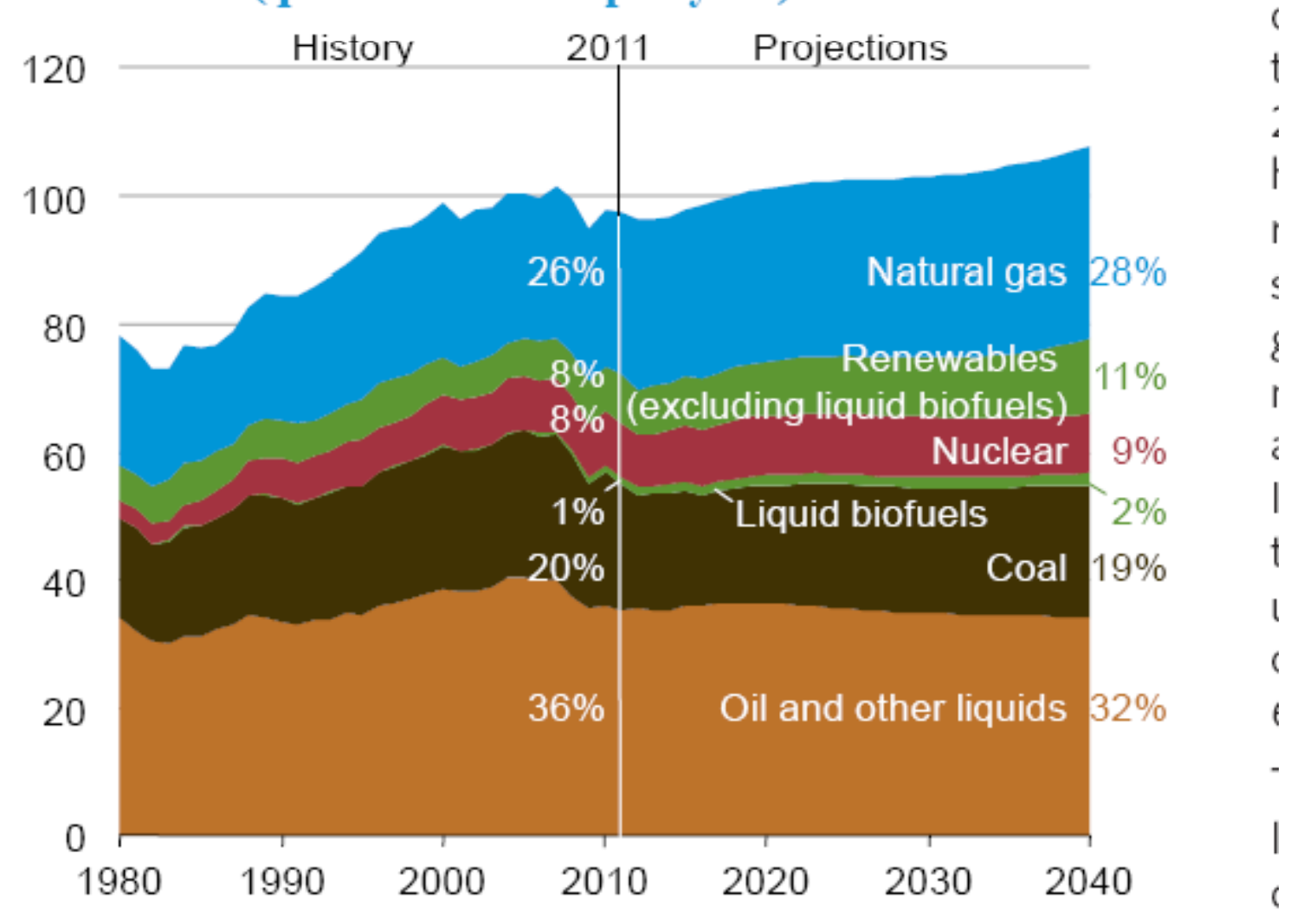

2013 AEO Early Release (December 2012) 
Figure 10. U.S. energy production by fuel, 1980-2040 (quadrillion Btu)

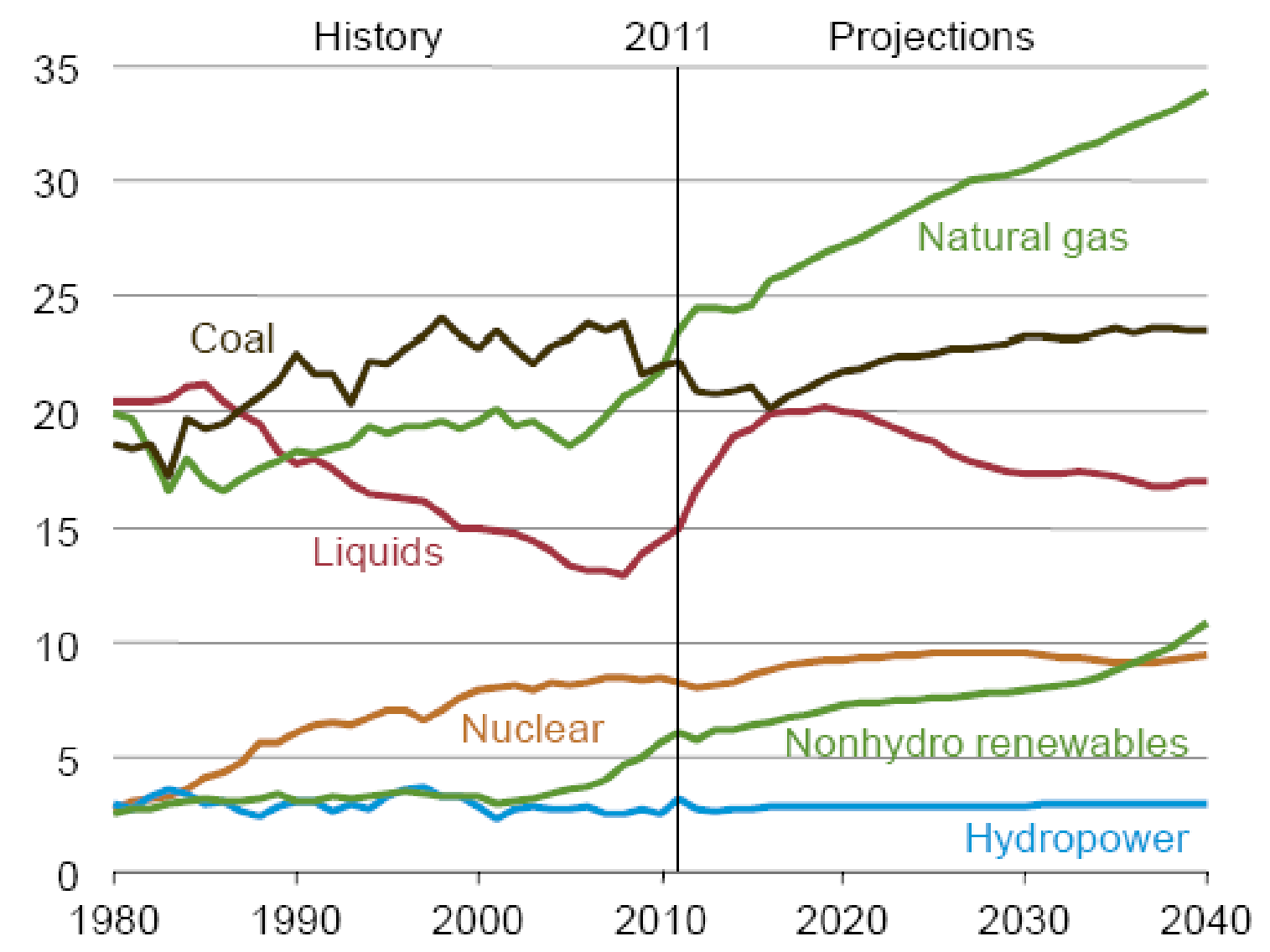


Figure 11. U.S. liquid fuels supply, 1970-2040 (million barrels per day)

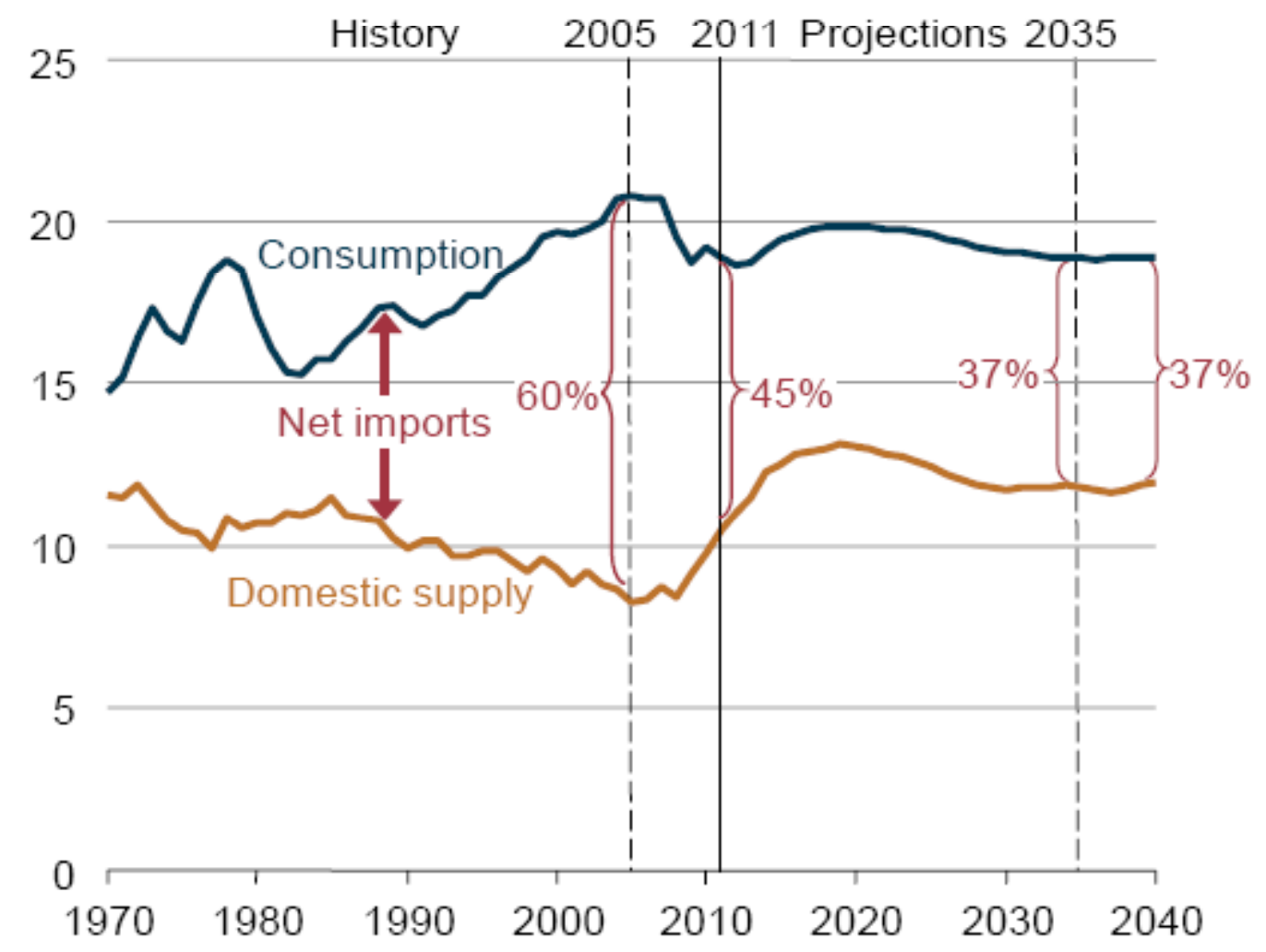

2013 AEO Early Release (December 2013) 
Figure 115. Carbon dioxide em issions by sector and fuel, 1990-2025 (million metric tons)

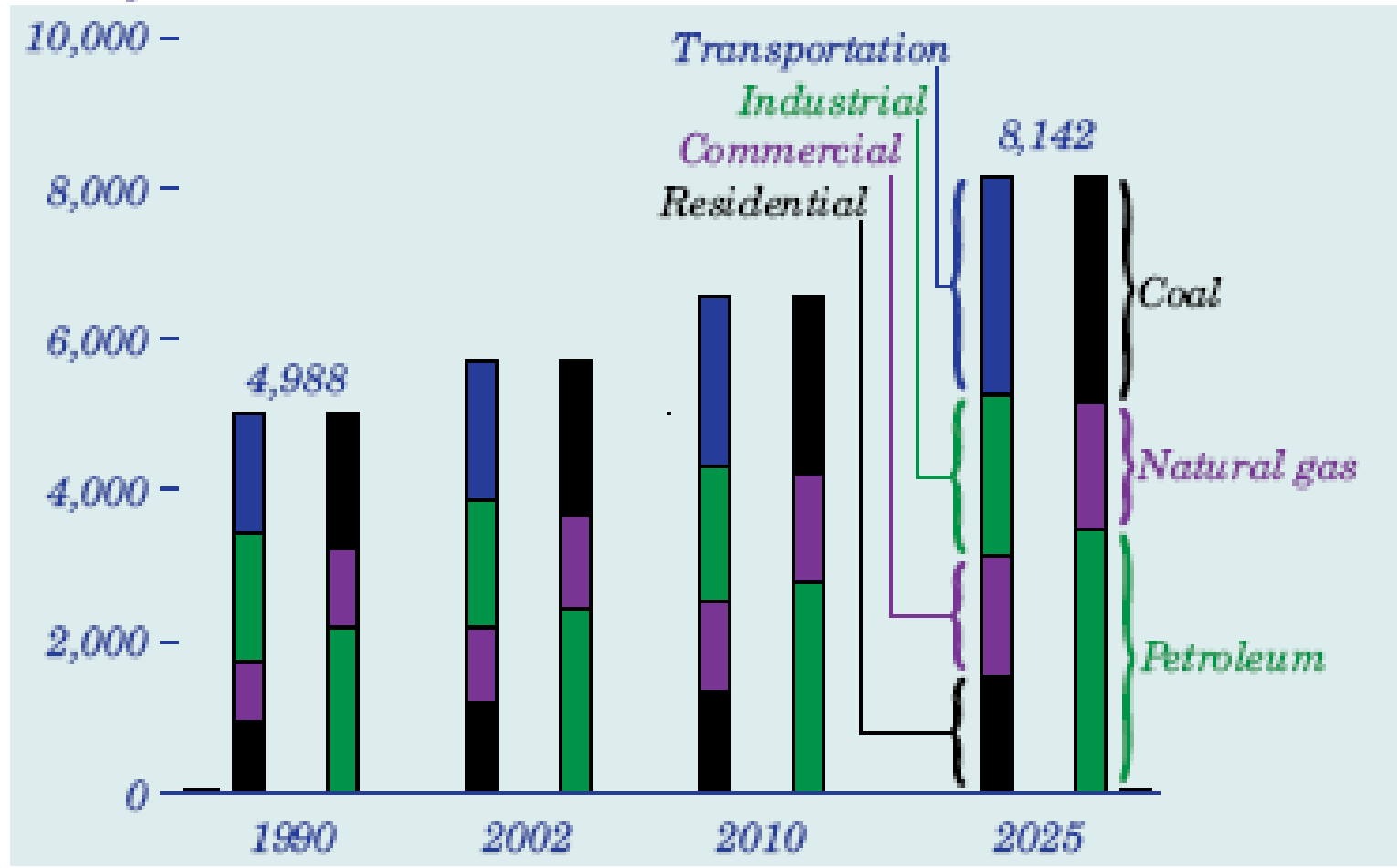

EIA AEO 2004 


\section{Projected energy-related carbon dioxide emissions remain below their 2005 level}

Figure 122. U.S. energy-related carbon dioxide emissions by sector and fuel, 2005 and 2035

(million metric tons)

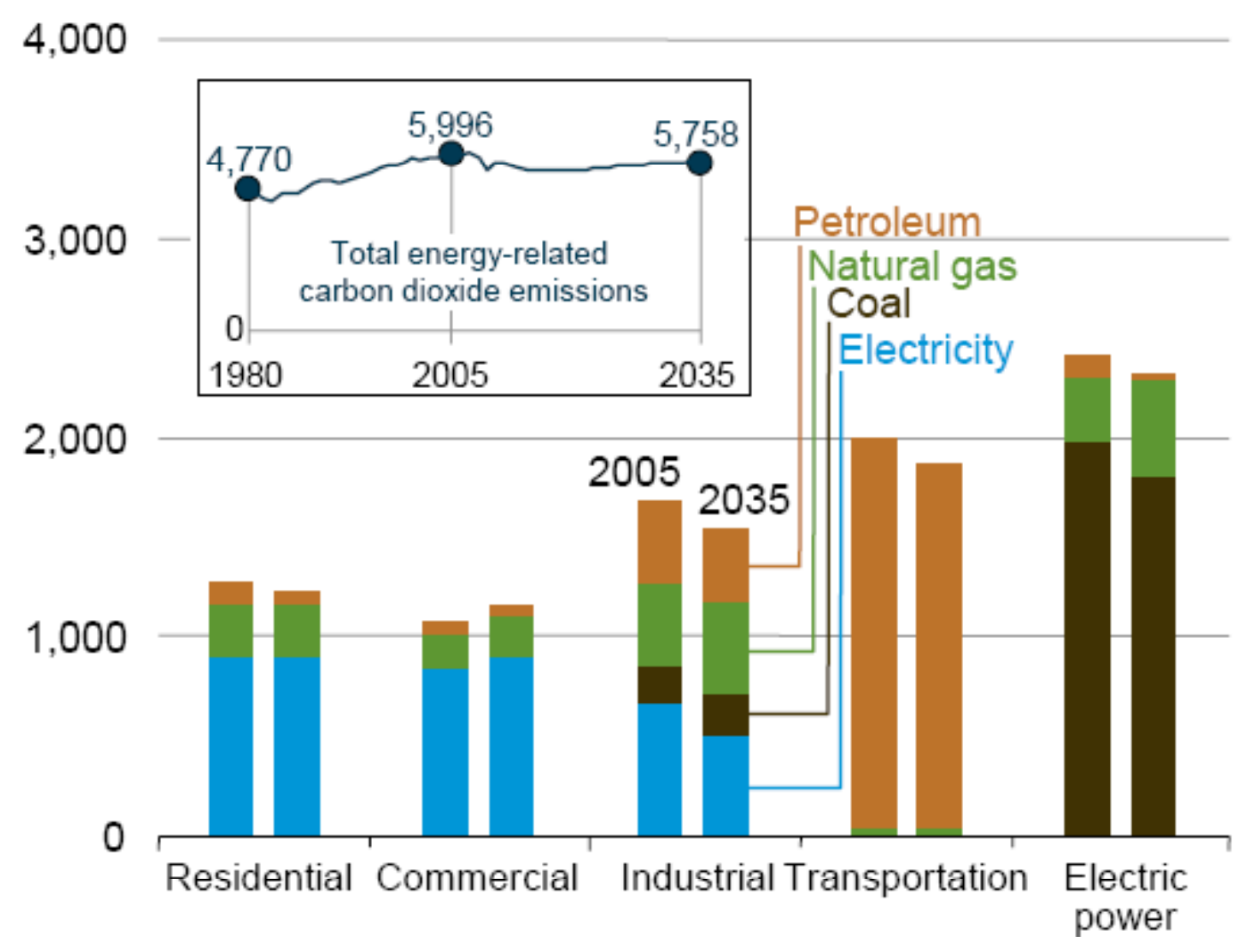

Source: EIA AEO 2012 
Figure 13. U.S. energy-related carbon dioxide emissions in recent $A E O$ Reference cases (percent change from 2005)

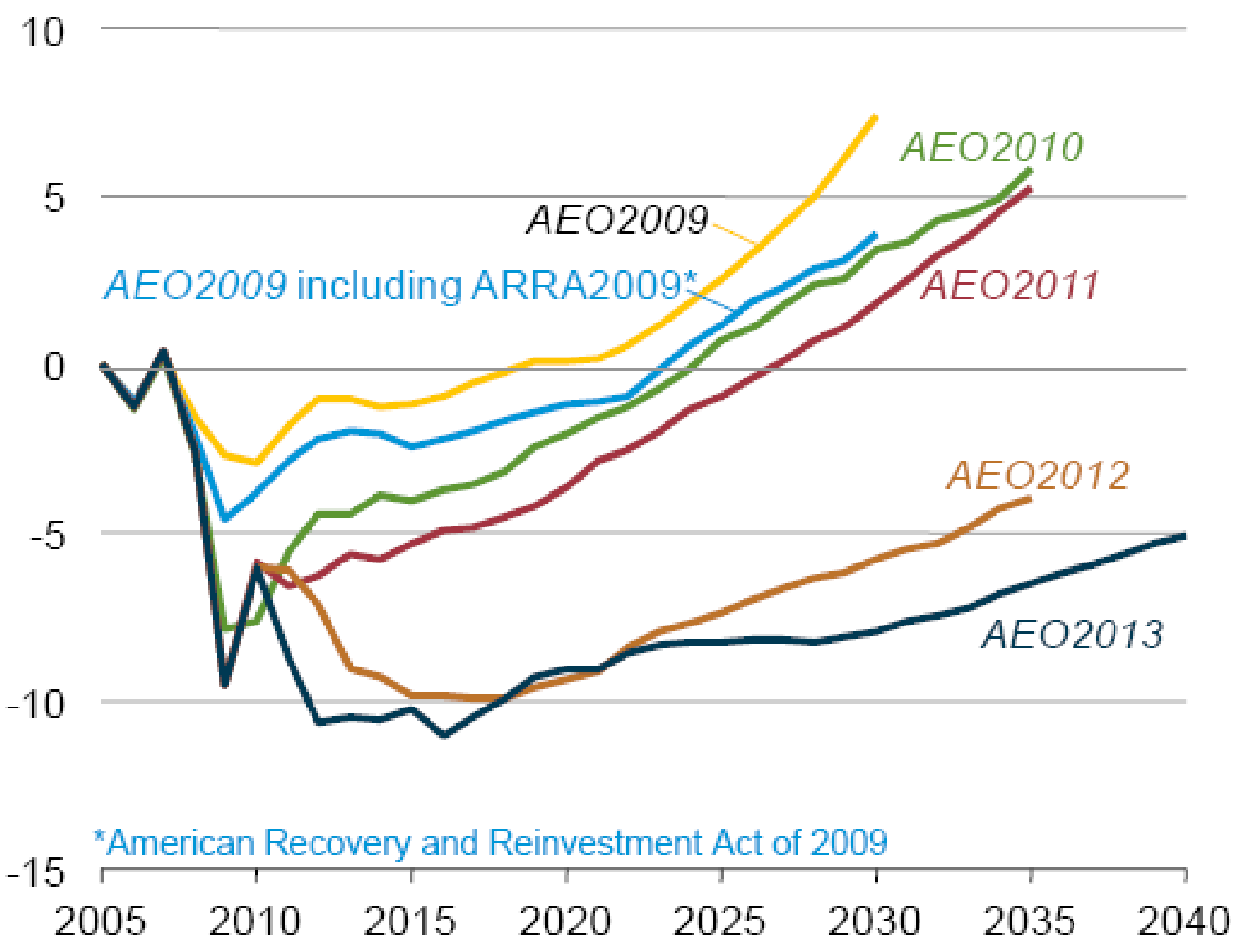

2013 AEO Early Release (December 2013) 
Figure 10: 2010 LNG Trade (Tcf)

\begin{tabular}{|c|c|c|c|c|c|c|c|c|c|c|c|c|c|}
\hline FromlTo & Africa & Canada & $\begin{array}{l}\text { China/ } \\
\text { India }\end{array}$ & $\begin{array}{c}\text { C\&S } \\
\text { America }\end{array}$ & Europe & FSU & $\begin{array}{c}\text { Korea/ } \\
\text { Japan }\end{array}$ & $\begin{array}{l}\text { Middle } \\
\text { East }\end{array}$ & Oceania & Sakhalin & $\begin{array}{c}\text { Southeast } \\
\text { Asia }\end{array}$ & U.S. & $\begin{array}{c}\text { Total } \\
\text { Exports }\end{array}$ \\
\hline Africa & & 0.03 & 0.05 & 0.31 & 1.33 & & 0.24 & 0.21 & & & 0.07 & 0.31 & 2.54 \\
\hline Canada & & & & & & & & & & & & & 0.00 \\
\hline China/India & & & & & & & & & & & & & 0.00 \\
\hline Europe & & & & 0.01 & 0.11 & & 0.05 & 0.01 & & & 0.00 & & 0.18 \\
\hline FSU & & & & & & & & & & & & & 0.00 \\
\hline Korea/Japan & & & & & & & & & & & & & 0.00 \\
\hline Sakhalin & & & 0.02 & & & & 0.39 & 0.00 & & & 0.02 & & 0.43 \\
\hline $\begin{array}{l}\text { Southeast } \\
\text { Asia }\end{array}$ & & & 0.14 & 0.06 & & & 1.92 & 0.01 & & & 0.21 & & 2.34 \\
\hline U.S. & & & & & & & 0.03 & & & & & & 0.03 \\
\hline $\begin{array}{l}\text { Total } \\
\text { Imports }\end{array}$ & 0.00 & 0.04 & 0.81 & 0.47 & 2.61 & 0.00 & 4.53 & 0.34 & 0.00 & 0.00 & 0.49 & 0.40 & 9.70 \\
\hline
\end{tabular}

Source: "The LNG Industry 2010," GIIGNL.

Global Production 120 Tcf

NERA Economic Consulting (2012) 
Figure 16: Projected Wellhead Prices (2010\$/MMBtu)

\begin{tabular}{|l|c|c|c|c|c|c|}
\hline & $\mathbf{2 0 1 0}$ & $\mathbf{2 0 1 5}$ & $\mathbf{2 0 2 0}$ & $\mathbf{2 0 2 5}$ & $\mathbf{2 0 3 0}$ & $\mathbf{2 0 3 5}$ \\
\hline Africa & $\$ 1.75$ & $\$ 1.89$ & $\$ 2.09$ & $\$ 2.31$ & $\$ 2.55$ & $\$ 2.81$ \\
\hline Canada & $\$ 3.39$ & $\$ 3.72$ & $\$ 4.25$ & $\$ 5.20$ & $\$ 5.64$ & $\$ 6.68$ \\
\hline China/India & $\$ 12.29$ & $\$ 12.86$ & $\$ 13.00$ & $\$ 13.25$ & $\$ 13.57$ & $\$ 13.51$ \\
\hline C\&S America & $\$ 2.00$ & $\$ 2.16$ & $\$ 2.39$ & $\$ 2.64$ & $\$ 2.91$ & $\$ 3.22$ \\
\hline Europe & $\$ 9.04$ & $\$ 9.97$ & $\$ 10.80$ & $\$ 11.95$ & $\$ 12.39$ & $\$ 13.23$ \\
\hline FSU & $\$ 4.25$ & $\$ 4.60$ & $\$ 5.08$ & $\$ 5.61$ & $\$ 6.19$ & $\$ 6.84$ \\
\hline Korea/Japan & $\$ 14.59$ & $\$ 15.30$ & $\$ 15.47$ & $\$ 15.79$ & $\$ 16.19$ & $\$ 16.11$ \\
\hline Middle East & $\$ 1.25$ & $\$ 1.35$ & $\$ 1.49$ & $\$ 1.65$ & $\$ 1.82$ & $\$ 2.01$ \\
\hline Oceania & $\$ 1.75$ & $\$ 1.89$ & $\$ 2.09$ & $\$ 2.31$ & $\$ 2.55$ & $\$ 2.81$ \\
\hline Sakhalin & $\$ 1.25$ & $\$ 1.35$ & $\$ 1.49$ & $\$ 1.65$ & $\$ 1.82$ & $\$ 2.01$ \\
\hline Southeast Asia & $\$ 2.00$ & $\$ 2.16$ & $\$ 2.39$ & $\$ 2.64$ & $\$ 2.91$ & $\$ 3.22$ \\
\hline U.S. & $\$ 3.72$ & $\$ 3.83$ & $\$ 4.28$ & $\$ 5.10$ & $\$ 5.48$ & $\$ 6.36$ \\
\hline
\end{tabular}

NERA Economic Consulting (2012) 
Figure 4.3 $\triangleright$ Indicative economics of LNG exports from the United States

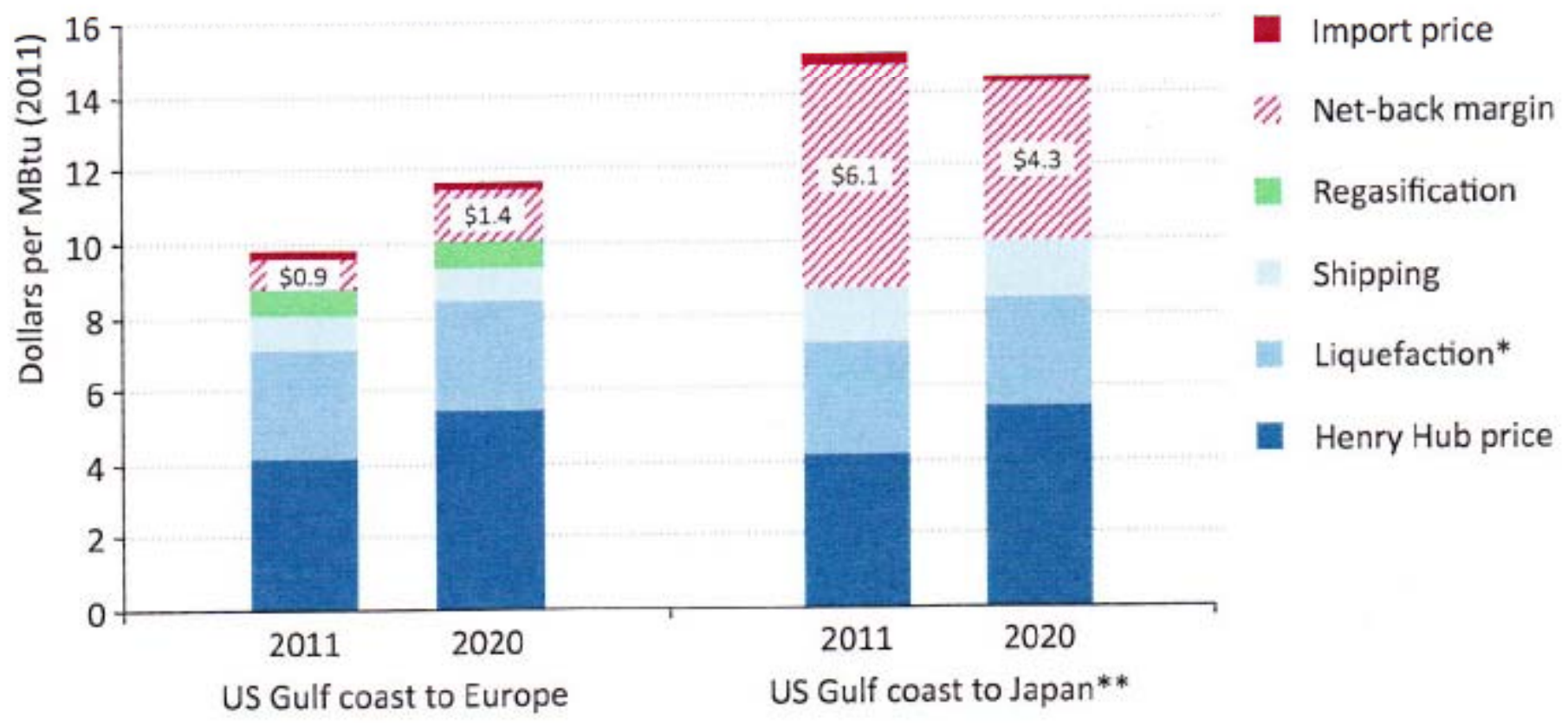

* Includes cost of pipeline transport to export terminal. ** Widening of the Panama Canal, due to be completed in 2014, will allow for more LNG tanker traffic.

Notes: LNG costs are levelised assuming asset life of 30 years and a $10 \%$ discount rate. The Japanese import price is for liquefied gas, so it does not include regasification. 
Figure 7: Comparison of EIA and NERA Maximum Wellhead Price Increases

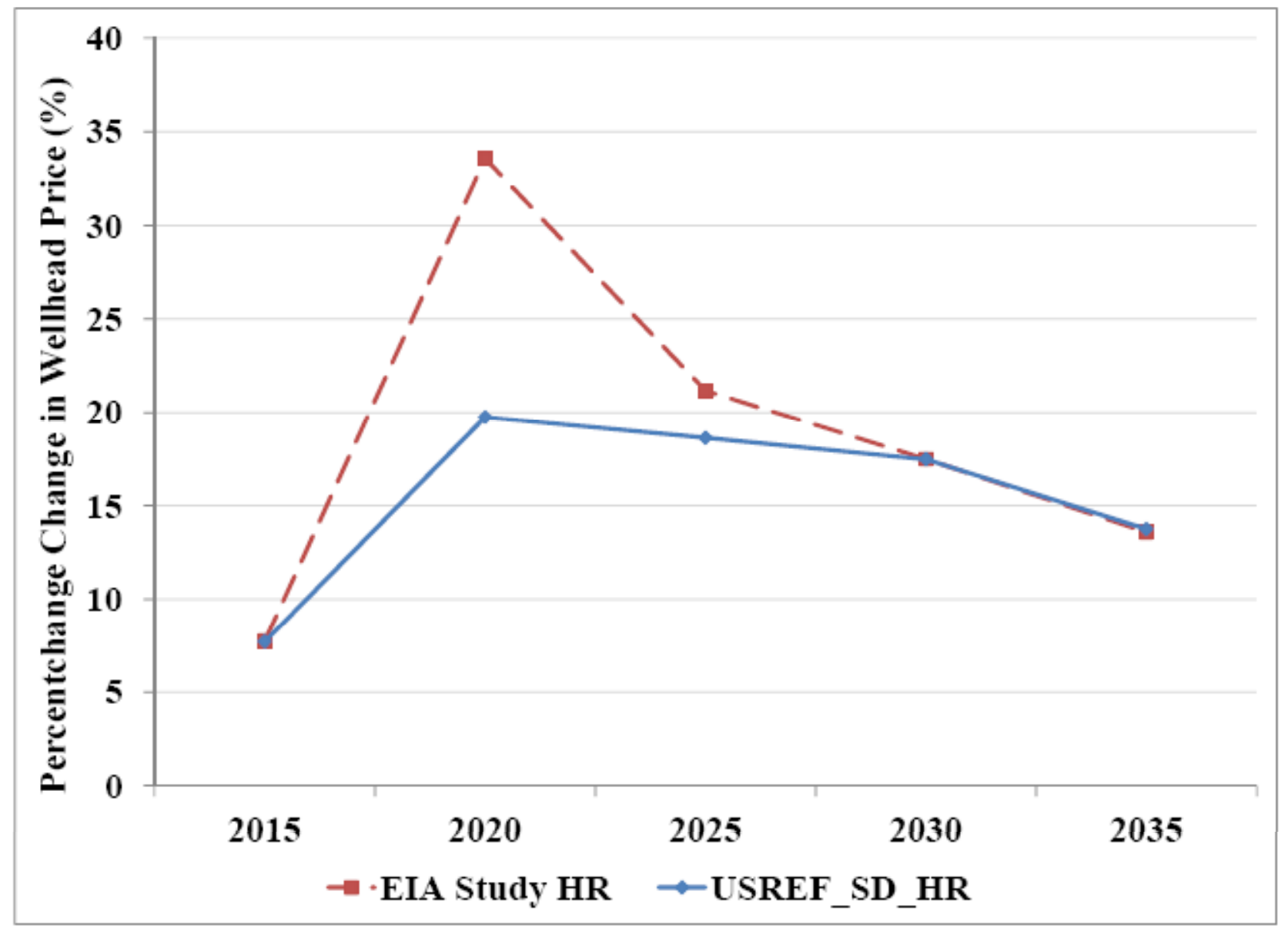

NERA Economic Consulting (2013) 


\section{Environmental Issues}

- Does "fracking" lead to contamination of drinking water aquifers with methane?

- Hydraulic fracturing has been used in the oil and gas industry since at least 1947 and probably earlier

- Fracking in shale deposits per se is unlikely to lead directly to drinking water contamination because it takes place thousands of feet below water aquifers

- If there is contamination of drinking water it is much more likely due to failures in the vertical well casing, stray gas releases from conventional pools during vertical drilling, or surface spills of fracking fluids and drilling mud

- The connection between shale gas production and groundwater contamination is weak and the studies that claim to document a connection are deficient --- little if any "before and after" analysis 


\section{Environmental Issues}

- There are more important pathways leading to potential environmental impacts that are probably of more concern

- Vertical well failures

- Above ground spills of toxic fracking fluids and drilling mud and inadequate long-term disposal methods

- Air emissions from drilling and support equipment

- Increased truck traffic delivering equipment and disposing of wastes

- Fresh water use

- Earth tremors from below-ground disposal of fluids

- Methane emissions

- Regulatory frameworks and capabilities vary widely from state to state and between states and federal government

- Best practices for regulatory frameworks have not been developed

- Lack of transparency by the industry has created suspicions that environmental impacts are not being taken into account adequately 